\title{
Colorectal Surgery and Anastomotic Leakage
}

\author{
P.B. Soeters ${ }^{a}$ J.P.J .G.M. de Zoete ${ }^{a}$ C.H.C. Dejong a N.S. Williams ${ }^{b}$ \\ C.G.M.I. Baeten ${ }^{a}$ \\ aDepartment of Surgery, University Hospital Maastricht, The Netherlands and ${ }^{b}$ Department of Surgery, \\ The Royal London Hospital, London, UK
}

\section{Key Words}

Colorectal surgery · Anastomotic leakage · Risk factors .

Treatment · Primary anastomosis · Hartmann's

procedure - Diverting colostomy - Staged procedures .

Abscess drainage $\cdot$ Disconnection anastomosis

\section{Introduction}

Anastomotic leakage after colorectal surgery occurs in $5-15 \%$ of patients undergoing this type of surgery and leads to a substantial morbidity and mortality. Many factors determine the occurrence of anastomotic leakage and its sequelae, including both patient- and surgery-related factors.

In the literature, much emphasis is put upon the type and quality of surgery responsible for complications. However, many patient-related factors have enormous bearing upon the occurrence of anastomotic leakage, as well as on the severity of the complication. The health state of the patient substantially determines the severity of morbidity and mortality rate. In this paper we will therefore first discuss risk factors for anastomotic leakage, and for survival after leakage. Subsequently, we will dis- cuss the type of surgery that may be undertaken in view of these risk factors. Finally, we will discuss a treatment approach to anastomotic leakage.

\section{Risk Factors for Septic Complications}

\section{Blood Loss}

Blood loss during operation is a risk factor for anastomotic healing. In a recent study, evidence was put forward that substantial blood loss during operation explained most of the variance in outcome and the occurrence of complications [1]. This is very likely due to the blood transfusions administered and to their immunosuppressive effect. In other reports the same finding is described in relation to the occurrence of post-operative complications. This untoward side effect of blood transfusions has even been tried to be used to the advantage of patients by administering blood prior to organ transplants. In today's practice these findings have led to a more conservative approach with regard to the replenishment of blood. If patients have good cardiac and oxygenating capacity, a haemoglobin level $>4.5$ or $5.0 \mathrm{mmol} / 1$ can safely be accepted.

\section{KARGER \\ Fax +4161306 1234 \\ E-Mail karger@karger.ch \\ www.karger.com

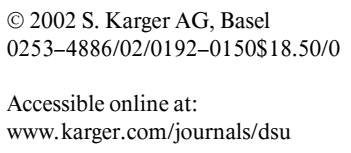

P.B. Soeters

University Hospital Maastricht

PO Box 5800

NL-6202 AZ Maastricht (The Netherlands)

Tel. +31 43 3876543, Fax +31 43 3875473, E-Mail pb.soeters@ah.unimaas.nl 


\section{Weight Loss}

Nutritional state may represent a risk factor for anastomotic leakage. As early as 1935 , Studley reported an increased surgical mortality in benign peptic ulcer disease when patients had lost more than $10-15 \%$ of their body weight in the 6 months before operation. This finding was confirmed repeatedly in later years. Especially above a weight loss of $15 \%$, mortality rises exponentially. There are reports in the literature that a pre-operative refeeding course of 7-10 days may improve outcome and diminish complications. This can only safely be performed when patients are not obstructed or infected. Patients with gastric outlet obstruction, for instance, run the risk of aspiration pneumonia, which increases the risk of serious complications even more than the malnourished state itself. Patients with necrotizing or penetrating tumours leading to abscess formation often suffer from severe inflammatory activity and do not benefit from a course of pre-operative nutritional support. Such patients should therefore be operated without much delay. This statement does not rely on randomized studies but rather on the fact that during severe ongoing inflammation the benefit of nutritional support is dubious, and on the generally accepted clinical experience that the longer necrotizing tumour abscesses are allowed to progress, the worse the outcome will be.

\section{Cardiorespiratory Co-Morbidity}

Ansari et al. [2] found that cardiorespiratory diseases had a negative effect on outcome in patients undergoing surgery for colorectal disease. In the intensive care the patients dying from severe septic surgical complications are those that are not able to generate a hyperdynamic circulation and to meet increased metabolic demands during sepsis. To fight sepsis, patients need to increase cardiac output for days to weeks to ascertain substrate and oxygen delivery to the tissues in a situation where tissue extraction of these compounds is compromised. If the patient is suffering from severe cardiac pump failure, these demands cannot be met leading to an increase in mortality. Mutatis mutandis - this also applies to patients with modest or no respiratory reserve. Both as a consequence of general anaesthesia and abdominal pain, precluding normal breathing, a certain degree of atelectasis is usually present following surgery and increases demands on both the respiratory muscle pump and on the capacity of alveolar diffusion of oxygen and carbon dioxide. In cachectic patients suffering from COPD, these capacities are limited leading to the need for artificial ventilation especially in the face of sepsis. This in turn also leads to secondary complications like ventilator-associated pneumonia's and an increase in mortality rate.

\section{Liver Failure}

It is well recognized that chronic liver insufficiency is associated with an overall high complication rate for infection and disturbed wound healing during any disease or surgical intervention. Liver disease itself does not preclude surgery but rather the remaining liver function determines the ability to withstand sepsis [3]. The liver is the largest 'immune organ' in the body and the inability of this organ to properly respond to the increased metabolic demands of sepsis and trauma, precipitates mortality [3].

\section{Intoxication}

Sorensen et al. [4] reported that smoking and a substantial alcohol intake coincided with an increase in morbidity and mortality after surgery. The mechanisms involved are not clear, but the pulmonary epithelial damage inflicted by smoking may precipitate invasive lung infection. Alcohol may induce complications via its damaging effects on liver function, but it is not certain to what extent there is a direct toxic effect.

\section{Local Situation}

Finally and most importantly, the MPI index [5] or Hinchey stage [6] have been found to be significant predictors of morbidity and mortality. The MPI index considers several patient-related factors and severity of disease, adding up to a score. Hinchey stage depends on the severity of local infection, ranging from contained intramesocolic or extra-mesocolic abscess formation to faecal peritonitis. The more diseased and the more septic the patient is on admission and at operation (high MPI index, high Hinchey stage), the more severe morbidity and the higher mortality is. There are several factors contributing to these facts, none of which are clearly quantified in prospective randomized investigations. Much 'collateral' evidence however supports these statements that intuitively will also be supported by clinicians. It is a general clinical finding that wounds do not heal well in septic patients, hairs do not grow well, and granulation tissue is not healthy. On the basis of this 'collateral evidence' it may be assumed that anastomotic healing is compromised as well. Another 'fact of clinical experience' is that for good anastomotic healing, a healthy surrounding matrix is necessary (absence of abscesses or haematoma, normal bowel, omentum, etc.). Most clinicians would therefore hesitate to construct a primary anastomosis in the presence of faecal peritonitis. This 'working hypothesis' also is not 
Table 1. Level of evidence and grade of recommendation

\begin{tabular}{lll}
\hline Level of evidence & Grade of recommendation \\
\hline Level I & $\begin{array}{l}\text { Randomized trial in seriously ill patients with low risk of error, } \\
\text { i.e., blinded, objective criteria, intention-to-treat analysis }\end{array}$ & $\begin{array}{l}\text { Grade A: } \\
\text { Supported by level I evidence }\end{array}$ \\
\hline Level II & $\begin{array}{l}\text { Randomized trial in seriously ill patients with high risk of error, } \\
\text { i.e., not blinded, objective criteria not used, } \\
\text { non-intention-to-treat analysis }\end{array}$ & $\begin{array}{l}\text { Grade B: } \\
\text { Supported by at least one level II study }\end{array}$ \\
\hline Level II & $\begin{array}{l}\text { Nonrandomized trial of seriously ill patients or randomised } \\
\text { trial of non-seriously ill patients or randomized trial of seriously } \\
\text { ill patients measuring surrogate outcomes }\end{array}$ & $\begin{array}{l}\text { Grade C: } \\
\text { No support from level I or II studies }\end{array}$ \\
\hline Level IV & $\begin{array}{l}\text { Nonrandomized trial in non-seriously ill patients or animal } \\
\text { studies or biological rationale }\end{array}$ & No recommendation \\
\hline
\end{tabular}

Table 2. Results of search query PubMed

\begin{tabular}{llll}
\hline Search query PubMed & $\begin{array}{l}\text { Matching } \\
\text { Articles }\end{array}$ & $\begin{array}{l}\text { Randomized } \\
\text { controlled } \\
\text { trials }\end{array}$ & $\begin{array}{l}\text { Level I } \\
\text { and II }\end{array}$ \\
\hline $\begin{array}{l}\text { Hartmann and anastomosis } \\
\begin{array}{c}\text { Colonic resection and } \\
\text { primary anastomosis }\end{array}\end{array}$ & 287 & 6 & 2 \\
\hline
\end{tabular}

substantiated by solid evidence. Consequently, the 'metaanalysts' will question such statements.

\section{Type of Surgery}

Clearly, there is not much discussion that resection and primary anastomosis can be performed in most patients without significant co-morbidity undergoing elective colorectal surgery above the promontorium. Whether a diverting loop ileostomy or colostomy should be performed in all patients undergoing elective low anterior resection is an issue under ongoing debate, especially since the advent of the TME procedure. The picture becomes progressively blurred once general patient-related factors or local infectious problems come into play. However, in the modern literature a trend can be observed towards more definitive surgery. Resection and primary anastomosis has been claimed to be feasible even if complicated [7-9]. Thirty to 20 years ago, primary resection was dissuaded under such circumstances. Drainage and a diverting proximal loop colostomy was advocated. More recently in most instances primary resection has been advocated either with primary anasto- mosis and diverting colostomy or with proximal end colostomy and distal closure of the rectal stump (Hartmann's procedure). At present, especially the younger generation of surgeons promote resection and primary anastomosis on the basis of the claim that this is feasible and that delayed procedures prolong the disease period and the period of debilitation and that the secondary surgical procedures are also fraught with complications.

In the past decade in the English literature at least 700 publications have appeared on the subject of the prevention of septic complications or of their treatment following colorectal surgery. It also has become very popular to act in clinical practice on the basis of evidence-based medicine. For this purpose it is necessary to scrutinize the literature and to construct a meta-analysis of available evidence. In principle, publications are reviewed and graded on the basis of their quality as level I-II evidence (table 1). Level I evidence is acquired when a clinical study is prospective, randomized and double-blinded, and when endpoints are clinical regarding morbidity, mortality and length of stay in the hospital (LOS). Level IV evidence does not contain any of these characteristics. Level I evidence furnishes a strong recommendation to follow the result of the outcome of the study. Level IV evidence does not furnish true recommendations. A metaanalysis collects the results of the available studies on the basis of their quality and determines the risk ratios of the different surgical approaches. We have tried to perform an analysis of the available literature regarding resection and primary anastomosis versus delayed procedures (table 2). We defined out of 680 studies no level I studies and only four level II studies, none of them leading to a Grade $\mathrm{B}$ or $\mathrm{C}$ recommendation (table 3). The main reason for the 
Table 3. Level II studies

\begin{tabular}{|c|c|c|c|c|}
\hline $\begin{array}{l}\text { Group } \\
\text { (first author) }\end{array}$ & Patients & Comparison & Benefit & $\begin{array}{l}\text { Level of } \\
\text { evidence }\end{array}$ \\
\hline Burke [12] & 186 & Bowel preparation versus no bowel preparation & No & II \\
\hline Kronborg [13] & 62 & $\begin{array}{l}\text { Acute transverse colostomy, suture and omental covering of a } \\
\text { visible perforation versus acute resection without primary } \\
\text { anastomosis }\end{array}$ & Yes & II \\
\hline Merad [14] & 494 & The benefit of pelvic drains versus their potential harm & No & II \\
\hline Merad [15] & 705 & $\begin{array}{l}\text { Omentoplasty versus no omentoplasty in the prevention of } \\
\text { anastomotic leakage }\end{array}$ & No & II \\
\hline
\end{tabular}

low level of evidence is the lack of randomization. The choice of the procedure therefore is very likely made on intuitive choices made by the surgeon on the basis of the degree of local or generalized peritonitis and possibly also on the basis of the condition and co-morbidity of the patient [10]. The similar results of primary anastomosis after resection and the shorter LOS are most likely due to the fact that the patients undergoing this procedure had less severe peritonitis and were in a better condition.

\section{Size of the Insult versus Host Defence}

It is obvious that resection and primary anastomosis is the approach of choice if no septic complications occur. Even in patients with local or moderately generalized peritonitis, uneventful recovery may take place in a considerable proportion of patients. The more serious the peritonitis, the more likely it is that septic complications will occur. This risk can be taken if the patient is judged to be able to respond adequately and consequently to survive a septic episode. Of all patients in good condition a few will therefore have more serious complications and will need long-term secondary treatment but a substantial proportion will benefit because they will quickly recover after a one-stage procedure. If this approach is taken in the patients with serious risk factors (see in subsection Risk Factors), the patients that develop septic complications will have a much higher risk of dying. This patient group should therefore be treated more defensively. Limited surgery should be accomplished without risky anastomoses in an infected area. The size of the insult should be tailored to the ability of the patient to raise an adequate host defence. This not only applies to the operation insult itself but also to the potential complication that may arise. Everything should be done to limit the risk of septic complications in patients with for instance chronic cardiac, respiratory, hepatic failure. On the basis of these considerations, not one surgical approach can be advocated as being superior to others. Instead, the surgical approach should be tailored to the health of the patient.

\section{Management of Leakage}

The first subsections of this paper have focussed on prevention of leakage and on the limitation of mortality by choosing a surgical approach intended to prevent leakage in patients that would not survive severe septic complications. However, once leakage has occurred, a concerted effort should be undertaken to limit morbidity and mortality and to effectively treat the leak and the resulting sepsis. Several aspects need detailed attention to achieve an optimal result.

Luna-Perez et al. [11] reviewed 13 patients with colorectal leakage and found that early diagnosis and treatment were crucial in obtaining optimal results. Wounds draining sero-sanguinolent fluid or overt pus, adynamic ileus, pain, malaise, no stool passage, fever and leukocytosis should all raise the suspicion of anastomotic leakage. Cardiorespiratory complications in the first 7-10 days following colonic surgery should also raise the suspicion of a leak. Early diagnosis will allow early intervention, in turn minimizing the morbidity and mortality of the surgical complication.

A tailored approach is necessary to abscess drainage.

(1) Some patients have only limited leakage with small adjacent abscesses revealed by enemas to opacify the anastomosis. Clinical signs are often mild including slight fever and malaise, modest abdominal discomfort and normal vital signs. In such patients a conservative attitude of wait and see appears to be warranted. Only when clinical 
signs deteriorate is a more aggressive approach necessary.

(2) Small lateral anastomotic failure with adjacent unilocular abscess of greater size (approx. $5 \mathrm{~cm}$ diameter or larger) generally needs drainage either internally via the anastomosis, or externally via CT scan-guided puncture techniques or small surgical incisions. Such leakages may sometimes require a diverting loop ileostomy or colostomy.

(3) If anastomotic failure amounts to half or more of the circumference of the anastomosis, the likelihood of spontaneous closure is small. Under these circumstances a Hartmann procedure is required.

(4) In case of multilocular abscesses and abdominal spread laparotomy and abdominal debridement is generally necessary, often accompanied by a Hartmann procedure. In these severely ill patients it is not always easy to establish whether there is a true anastomotic disruption during the operation. A more aggressive approach appears to be warranted. In case of doubt it can be considered ultimately the safest approach to disconnect the anastomosis. In this condition the first concern should be to treat sepsis effectively and to reduce mortality.

Once a leakage and the resulting abscess have been successfully treated and the patient has become metabolically stable, the anastomotic defect may heal quickly or may develop into a faecal fistula. Some of these faecal fistulas may heal spontaneously when the situation is favourable.

(1) The defect in the anastomosis should be lateral and relatively small and comprise substantially less than 50\% of the circumference.

(2) The edges of the anastomosis should be healthy and vascularly well perfused, whereas neighbouring abscesses should be adequately drained. For this purpose, drainage should be continued for weeks depending on drain production. When well-draining tracts have developed into mature fistulae, withdrawal of the drain may be tried to close the fistula spontaneously.

(3) This is only possible when distal passage of stool is uninhibited, which may be enhanced when stool passage is diminished by proximal diverting enterostomies. Another method to achieve this is to drain the colon or rectum distal to the leak by inserting a rectal tube. This diminishes the pressure in the area of the defect, which may promote its closure. When circumstances are favourable, spontaneous closure occurs in more than $50 \%$ of cases. There is no evidence in the literature that 'starvation of the lower gut' induced by parenteral nutrition may help to close the fistula. Stoma-like fistulae in open wounds do not close spontaneously and need to be closed surgically.
(4) Any attempt at operative repair should be undertaken when patients have become metabolically stable, have regained an optimal condition and when inflammatory signs have subsided. For this purpose, plasma albumin, haemoglobin, sedimentation rate and C-reactive protein levels may be used as indicators. Re-operation should generally be postponed at least until 6 weeks after the last operation. This period is necessary for maturation of adhesions, the disappearance of wound oedema and consequently the accessibility of the surgical area.

\section{Conclusions}

There is not one 'best' approach to colorectal leakage and the resulting morbidity and mortality. Several risk factors exist which either increase the rate of anastomotic leakage or which preclude an adequate response to complications. Patients suffering from these risk factors (chronic organ disease, malnutrition and blood loss) should undergo the safest and least risky procedures. In low-risk patients riskier procedures may be undertaken because these patients will also survive leakage, if quickly and adequately treated. In this last patient group, resection and primary anastomosis will accelerate recovery whereas complications induced by the riskier procedure will not substantially increase mortality.

The answer how leakage should be treated, specifically whether stomata should be constructed and how abscesses should be drained, depends on the degree of anastomotic failure and on the extent of abscess formation and faecal peritonitis. When $50 \%$ of the anastomosis is disrupted, the anastomosis should be dismantled. When abscess formation is extensive or when a faecal peritonitis is present, dismantling should also be considered and even more so in high-risk patients. 


\section{References}

1 Von Meyenfeldt MF, Soeters PB: Risk factors for post-operative complications. $\mathrm{Br} \mathrm{J}$ Clin Pract Suppl 1988;63:49-52.

2 Ansari MZ, Costello AJ, Ackland MJ, et al: Inhospital mortality and associated complications after bowel surgery in Victorian public hospitals. Aust NZ J Surg 2000;70:6-10.

3 Clowes GH Jr, Hirsch E, George BC, et al: Survival from sepsis. The significance of altered protein metabolism regulated by proteolysis inducing factor, the circulating cleavage product of interleukin-1. Ann Surg 1985;202:446-458.

4 Sorensen LT, Jorgensen T, Kirkeby LT, et al: Smoking and alcohol abuse are major risk factors for anastomotic leakage in colorectal surgery. Br J Surg 1999;86:927-931.

5 Hansen O, Graupe F, Stock W: Prognostic factors in perforating diverticulitis of the large intestine (in German). Chirurg 1998;69:443449.
6 Schilling MK, Maurer CA, Kollmar O, et al: Primary vs. secondary anastomosis after sigmoid colon resection for perforated diverticulitis (Hinchey stage III and IV): A prospective outcome and cost analysis. Dis Colon Rectum 2001;44:699-705.

7 Biondo S: Role of resection and primary anastomosis of the left colon in the presence of peritonitis. Br J Surg 2000;87:1580-1584.

8 Wedell J, Bauzhaf G, Chaoui R, et al: Surgical management of complicated colonic diverticulitis. Br J Surg 1997;84:380-383.

9 Gooszen AW, Gooszen HG, Veerman W, et al: Operative treatment of acute complications of diverticular disease: Primary or secondary anastomosis after sigmoid resection. Eur J Surg 2001;167:35-39.

10 Goyal A, Schein M: Current practices in leftsided colonic emergencies. A survey of US gastrointestinal surgeons. Dig Surg 2001;18:399_ 402.

11 Luna-Perez P, Rodriguez-Martinez HA, Chavez-Mercado L: Treatment of anastomotic leakage following low anterior resection for rectal adenocarcinoma. Rev Invest Clin 1999;51: 23.
12 Burke P, Mealy K, Gillen P, et al: Requirement for bowel preparation in colorectal surgery. $\mathrm{Br} \mathrm{J}$ Surg 1994;81:907-910.

13 Kronborg O: Treatment of perforated sigmoid diverticulitis: A prospective randomized trial. Br J Surg 1993;80:505-507.

14 Merad F, Hay JM, Fingerhut A, et al: Is prophylactic pelvic drainage useful after elective rectal or anal anastomosis? A multicenter controlled randomized trial. French Association for Surgical Research. Surgery 1999;125:529_ 535.

15 Merad F, Hay JM, Fingerhut A, et al: Omentoplasty in the prevention of anastomotic leakage after colonic or rectal resection: A prospective randomized study in 712 patients. French Association for Surgical Research. Ann Surg 1998;227:179-186.

16 Heyland DK: Enteral and parenteral nutrition in the seriously ill, hospitalised patient: A critical review of the evidence. J Nutr Health Aging 2000;1:31-41. 